\title{
Minimally symptomatic posterior reversible encephalopathy in Guillain-Barré syndrome
}

\author{
Giovanni Piccolo, MDa \\ Giuseppina Borutti, MD \\ Diego Franciotta, MD $^{\mathrm{a}}$ \\ Carla Arbasino, MD \\ Carlo Dallocchio, MD \\ Claudia Camana, MD \\ Giada Gola, MD' \\ Maria Grazia Egitto, MD ${ }^{c}$

\begin{abstract}
a C. Mondino National Neurological Institute, Pavia, Italy

${ }^{\mathrm{b}}$ Neurology Unit, Voghera Hospital, Voghera, Italy

' Radiology Unit, Voghera Hospital, Voghera, Italy
\end{abstract}

Correspondence to: Giovanni Piccolo

E-mail: giovanni.piccolo@mondino.it

\section{Summary}

Posterior reversible encephalopathy syndrome (PRES) is a rare occurrence in patients with GuillainBarré syndrome (GBS). Two patients with neuroradiological evidence of PRES without central nervous system (CNS) symptoms were recently reported.

We present the case of a GBS patient with minimal CNS symptoms and magnetic resonance imaging findings consistent with PRES.

PRES in GBS might be an underestimated condition. It should be suspected in GBS patients in the presence of even mild CNS symptoms. A timely PRES diagnosis along with early correction of autonomic system dysfunction in GBS patients is recommended to prevent possible dangerous CNS complications.

KEY WORDS: Guillain-Barré syndrome, magnetic resonance imaging, neurovegetative symptoms, posterior reversible encephalopathy syndrome

\section{Introduction}

Posterior reversible encephalopathy syndrome (PRES) is a central nervous system (CNS) disorder usually presenting with seizures, confusion, a decreased level of consciousness, headache and visual field defects. High blood pressure, autoimmune disorders, cytotoxic drugs, sepsis, pre-eclampsia or eclampsia and multiple organ dysfunction are the main causative or predisposing factors. Magnetic resonance imaging (MRI) findings mainly consist of white matter hyperintensities in the occipital lobes on T2-weighted images with features of vasogenic edema, sometimes involving the frontal and temporal lobes and the cerebellum (Fugate et al., 2010). There are recent reports of asymptomatic PRES, revealed by $\mathrm{MRI}$, associated with Guillain-Barré syndrome (GBS) (Parmentier et al., 2012; Barbay et al., 2013). Here we report the case of a patient who concurrently developed almost asymptomatic PRES and GBS.

\section{Case report}

Twenty days after a flu-like syndrome, a 58-year-old woman was admitted to a neurological unit because of progressive distal leg paresthesia and lower limb weakness which had begun seven days before her admission. Two days before admission, the weakness worsened and excessive daytime somnolence and a non-painful sense of head heaviness appeared.

At the age of 41 years, the patient had undergone bariatric surgery because of otherwise uncontrolled severe obesity. At the age of 56 she had developed type II diabetes mellitus, which was controlled by oral antidiabetic treatment. The general examination on admission showed an obese woman (weight: $89 \mathrm{~kg}$, height: $162 \mathrm{~cm}$, body mass index: $39 \mathrm{~kg} / \mathrm{m}^{2}$ ). Her blood pressure reading was $220 / 110 \mathrm{mmHg}$ and her heart rate was $110 \mathrm{bpm}$, rhythmic. When awake, she remained alert, well oriented and fully cooperative. Clinical assessment revealed normal cranial nerve function. In particular, visual acuity and visual field examination were normal. She showed symmetrical proximal limb weakness, more pronounced in the lower limbs (MRC grade $4 / 5$ and $2 / 5$ respectively), with absent tendon reflexes and reduced vibratory sense in the legs. Routine laboratory examination and arterial blood gas analysis were normal, with the exception of the finding of an increased blood sugar level (181 mg/dL, n.v. 65-110). Needle EMG examination was normal. Nerve conduction study showed bilaterally increased distal peroneal nerve and F-wave latencies, with motor unit potentials of reduced amplitude. Sural nerve conduction velocity and sensory potential amplitude were normal. Cerebrospinal fluid (CSF) examina- 
tion disclosed increased protein content $(109 \mathrm{mg} / \mathrm{dL}$, n.v. 10-30), with a normal cell count $(<2 / \mathrm{mmc})$. No oligoclonal bands were found and the search for herpes simplex, varicella zoster, Epstein Barr and cytomegalovirus DNA was negative. Persistent hypersomnolence prompted us to perform brain MRI, which showed white matter hyperintensities on FLAIR and T2-weighted images bilaterally involving the occipital and parietal lobes and, to a lesser degree, the frontal lobes, especially on the right side, without contrast enhancement. Diffusion-weighted images did not show areas of restricted signal but on the apparent diffusion coefficient (ADC) map cortical hyperintensities could be seen in the occipital lobes, related to vasogenic edema (Fig. 1A). Spinal cord MRI was normal. Blood pressure and heart rate were initially lowered by administration of urapidil chloride and beta blockers; thereafter, blood pressure was controlled with adjunctive angiotensin-converting enzyme inhibitors. During the first week following admission, signs and symptoms of intestinal obstruction developed. Direct abdominal X-rays, ultrasonography and colonoscopy did not show a mechanical obstruction, thus indicating paralytic ileus, which was resolved with administration of prokinetic drugs and neostigmine. Given the preservation of cranial nerve and respiratory function, we decided to treat the patient supportively, closely monitoring her neurological signs and respiratory function. Her neurological condition stabilized, and within two $\leq$ weeks a progressive improvement in strength began. At that time, brain MRI showed almost complete disappearance of the T2-weighted hyperintensities (Fig. 1B).

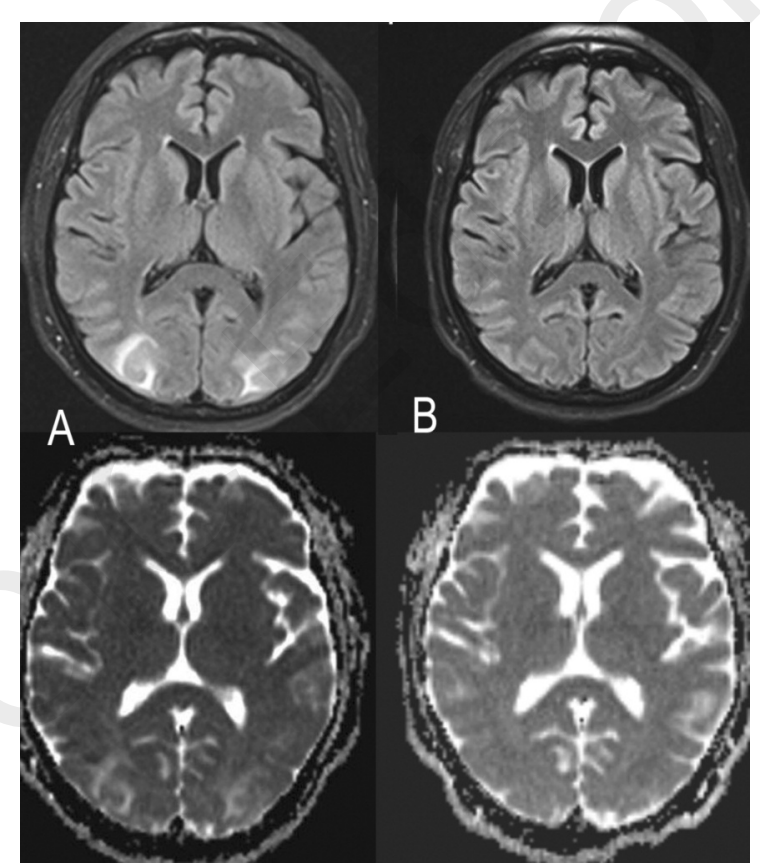

Figure 1 - Neuroradiological findings.

A) upper: axial FLAIR image shows white matter hyperintensities in both occipital lobes; lower: ADC map shows hyperintensities in both occipital lobes related to vasogenic edema.

B) axial FLAIR and ADC map, obtained after two weeks, show nearly complete disappearance of white matter hyperintensities.

\section{Discussion}

Our patient developed subacute tetraparesis with clinical, electrophysiological and CSF features that met the diagnostic criteria for GBS. However, the initial co-existence of subtle encephalopathic symptoms led us to suspect combined inflammatory involvement of the peripheral nervous system and the CNS and thus to perform neuroimaging studies. Unexpectedly, brain MRI showed neuroradiological features suggesting a diagnosis of PRES. Despite the limited motor involvement, the patient developed an autonomic system disturbance that included high blood pressure, sinus tachycardia and intestinal pseudo-obstruction. As recently reviewed (Rigamonti et al., 2012), PRES has, in rare cases, been reported both as an initial manifestation and a complication of GBS. The underlying pathophysiology is attributed mainly to altered cerebral blood flow autoregulation secondary to autonomic system dysfunction, probably along with cytokine-induced endothelial damage favoring blood-brain barrier disruption. In some patients with GBS or Miller-Fisher/Bickerstaff brain stem encephalitis syndrome, PRES has been interpreted as a complication of intravenous immunoglobulin (IVIg) treatment (Nakajima, 2005; Koichihara et al., 2008; Incecik et al., 2011; Stetefeld et al., 2014). In fact, GBS includes several clinical contingencies liable to promote the occurrence of PRES. Our case further supports the hypothesis that PRES in GBS patients may develop in the absence of prominent symptoms of CNS involvement and that it could represent an underestimated condition.

Accordingly, careful monitoring and timely correction of dysautonomic symptoms, even in non-critical GBS patients, could minimize the risk of PRES occurrence. On the other hand, even mild CNS symptoms occurring in GBS patients warrant attention, especially during administration of IVIgs, since a delay in PRES diagnosis and treatment could result in irreversible neurological complications (Yoon et al., 2013).

\section{References}

Barbay M, Arnoux A, Lebreton C, et al (2013). Clinically silent posterior reversible encephalopathy in Guillain-Barré syndrome. Can J Neurol Sci 40:267.

Fugate JE, Claassen DO, Cloft HJ, et al (2010). Posterior reversible encephalopathy syndrome: associated clinical and radiologic findings. Mayo Clin Proc 85: 427-432.

Incecik F, Hergüner MO, Altunbasak S, et al (2011). Reversible posterior encephalopathy syndrome due to intravenous immunoglobulin in a child with Guillain-Barré syndrome. J Pediatr Neurosci 6:138-140.

Koichihara R, Hamano S, Yamashita S, et al (2008). Posterior reversible encephalopathy syndrome associated with IVIG in a patient with Guillain-Barré syndrome. Pediatr Neurol 39:132-135.

Nakajima M (2005). Posterior reversible encephalopathy complicating intravenous immunoglobulins in a patient with Miller-Fisher syndrome. Eur Neurol 54:58-60.

Parmentier C, Vandermeeren Y, Laloux P, et al (2012). 
Asymptomatic posterior reversible encephalopathy revealed by brain $\mathrm{MRI}$ in a case of axonal Guillain-Barré syndrome. Clin Neurol Neurosurg 114: 1006-1009.

Rigamonti A, Basso F, Scaccabarozzi C, et al (2012). Posterior reversible encephalopathy syndrome as the initial manifestation of Guillain-Barré syndrome: case report and review of the literature. J Peripher Nerv Syst 17: 356360 .
Stetefeld HR, Lehmann HC, Fink GR, et al (2014). Posterior reversible encephalopathy syndrome and stroke after intravenous immunoglobulin treatment in Miller-Fisher syndrome/Bickerstaff brain stem encephalitis overlap syndrome. J Stroke Cerebrovasc Dis 23:e423-e425.

Yoon SD, Cho BM, Oh SM (2013). Clinical and radiological spectrum of posterior reversible encephalopathy syndrome. J Cerebrovasc Endovasc Neurosurg 15:206-213. 